\title{
Implementing Co-Regulated Feeding with Mothers of Preterm Infants
}

\author{
Suzanne M. Thoyre, PhD, RN, FAAN, \\ Frances Hill Fox Term Distinguished Professor, School of Nursing, The University of North \\ Carolina at Chapel Hill \\ Carol Hubbard, MSN, CPNP, \\ Coordinator, Special Infant Care Clinic, Division of Neonatal-Perinatal Medicine, University of \\ North Carolina Healthcare \\ Jinhee Park, PhD, RN, \\ Assistant Professor, Connell School of Nursing, Boston College \\ Karen Pridham, PhD, RN, FAAN, and \\ Professor Emerita, School of Nursing, University of Wisconsin-Madison \\ Anne McKechnie, PhD, RN, IBCLC \\ Assistant Professor, School of Nursing, University of Minnesota
}

\begin{abstract}
Purpose-The purpose of this study is to describe implementation of the Co-Regulated Feeding Intervention (CoReg), when provided by mothers and guided by intervention nurses trained in methods of guided participation (GP). CoReg aims to prevent stress during feeding and ease the challenge very preterm (VP) infants experience coordinating breathing and swallowing during the early months. Guided participation is a participatory learning method to guide the complex learning required of mothers.
\end{abstract}

Study Design and Methods-Sixteen mothers of 17 VP infants participated. Each mother received a median of five intervention sessions during the infant's transition to oral feeding. Intervention field notes, audio-recordings of the sessions, and video-recordings of the nurseguided feedings were reviewed, organized, and content analyzed to evaluate implementation.

Results-The co-regulated feeding intervention was well received by mothers; enrollment, participation, and retention rates were high. Most mothers chose to spread out the intervention sessions across the transition period. Scheduling sessions was the greatest barrier. Mothers had competing demands and infant readiness to eat could not be predicted. The top five issues identified as needing attention by the mother or nurse included reading cues, co-regulating breathing, providing motoric stability, regulating milk flow, and providing rest periods. Main GP strategies included joint attention with the mother to the dyad's feeding challenges, auditory assessment of breathing and swallowing, and reflection with planning for future feedings using video playback. 
Clinical Implications-Nurse presence while mothers feed affords rich opportunities to guide co-regulated, cue-based feeding. Co-Regulated feeding interventions would be enhanced if mothers are guided by the bedside nurse.

\section{Keywords}

Premature Infants; Feeding Methods; Maternal Behavior; Intervention Studies

\section{Introduction}

Very preterm infants (VP; $\leq 30$ weeks gestational age) gradually develop the ability to coordinate suck, swallow, breathe rhythms (Gewolb \& Vice, 2006), requiring more time than more mature infants to become full oral feeders (Dodrill, Donovan, Cleghorn, McMahon, \& Davies, 2008). As feeding skills are developing, VP infants demonstrate challenges marked by irregular respiration and apneic pauses during sucking bursts, alterations in sucking rate and strength in attempts to manage the flow of fluid, unstable oxygen saturation and heart rate, disengagement, and fatigue (Gewolb, Bosma, Reynolds, \& Vice, 2003; Hwang, Ma, Chen-Sea, Kao, \& Tsai, 2012; Mizuno et al., 2007).

Support of feeding skill development and protection from behavioral and physiologic stress during feeding may have significant implications for VP infants' future approach to eating. Feeding challenges continue into the post-discharge period at a high rate (Ross \& Browne, 2013) with one-third of VP infants reported to have feeding problems during the first year (DeMauro, Patel, Medoff-Cooper, Posencheg, \& Abbasi, 2011). Problems include: low appetite, oral-motor dysfunction, avoidance of feeding, vomiting, non-acceptance of textured foods, prolonged feeding times, oxygen desaturation during feeding, poor weight gain, and need for specialty feeding services (DeMauro et al., 2011; Jonsson, van Doorn, \& van den Berg, 2013; Migraine et al., 2013; Wang et al., 2010). Post-discharge, mothers also report challenges, including difficulty "reading" infant feeding cues, knowledge gaps, and discomfort with feeding (DeMauro et al., 2011; Reyna, Pickler, \& Thompson, 2006).

A co-regulated, cue-based approach to oral feeding has been proposed as effective for supporting the development of feeding skills of preterm infants (Kirk, Alder, \& King, 2007; McCain, Del Moral, Duncan, Fontaine, \& Pino, 2012; Puckett, Grover, Holt, \& Sankaran, 2008; Ross \& Philbin, 2011). Clinical guidelines for providing age-appropriate feeding of the premature and critically ill hospitalized infant are consistent with this effort in their recommendation to support infants' behavioral readiness, motoric stability, engagement, and stability of physiologic parameters during feeding (Sables-Baus et al., 2013).

Since VP infants' feeding skills are not yet mature at discharge, their mothers need to be adept at co-regulating feedings using the infants' cues to guide them. The learning curve for mothers can be steep as they report this is novel territory and can be complex (Stevens, Gazza, \& Pickler, 2014). We describe implementation of a method to guide mothers in providing co-regulated, cue-based feeding for their hospitalized VP infants.

To address the challenge of feeding for both the VP infant and his/her mother, a tested intervention, the Co-Regulated Feeding Intervention (CoReg) (Thoyre, Holditch-Davis, 
Schwartz, Melendez, \& Nix, 2012) was adapted to be delivered by mothers. The CoReg extends principles of cue-based feeding from assessment and response to learning to proactively structure feedings to prevent distress. Further, CoReg extends the definition of "cues" to include breathing and swallowing signals that dynamically change throughout feeding and therefore, provide important information on the infant's need for co-regulation.

Co-Regulated feeding interventions incorporate principles of age-appropriate care, including postural support, preparation of the infant with rooting, initiation of feeding based on infant readiness, minimization of movement throughout the feeding period, regulation of milk flow, and adding opportunities for breathing and rest. An innovative component of CoReg involves use of a small microphone placed on the infant's neck, which amplifies breathing and swallowing sounds to provide feedback to the feeder throughout the feeding. The initial development and efficacy testing of CoReg involved delivery of the intervention by specially trained nurses and feeding therapists (Thoyre, et al., 2012). This study demonstrated that CoReg-trained interventionists using the microphone system provided significantly more rest periods, began feedings more often with the infant in a state of readiness, stimulated the infant to suck less often, and tipped the bottle back more often to cue the infant to regulate breathing. During intervention feedings, there was more stability of the infant's oxygen saturation and heart rate, less disorganized behavior, less observable effort to breathe, and better swallowing organization (Thoyre, et al., 2012).

Two theories guided the extension of CoReg to an intervention delivered by mothers: dynamic systems theory explains emergence of the dyad's feeding behaviors and skills (Goldfield, 2007), and guided participation (GP) theory focuses on how to help a less experienced "other," in this case, the mother, adapt cognition and behavior to meet novel circumstances (Pridham, Limbo, Schroeder, Thoyre, \&Van Riper, 1998; Pridham \& Limbo, 2011). In this study, CoReg was delivered by mothers who were guided by a nurse trained in methods of GP. Guidance was individualized, based on the developing skills of the mother and her infant, to extend mothers' understanding of infant behavioral and physiologic cues, develop mothers' motor skills for provision of postural support and co-regulation of breathing, and develop mothers' ability to reflect on and adapt plans for future feedings (Thoyre, Park, Pados, \& Hubbard, 2013). Two innovations were used. First, using the microphone system, the nurse and mother simultaneously listened to the infant's amplified breathing and swallowing sounds throughout the feeding to jointly make feeding decisions as the mother fed. Second, the videotaped feeding was replayed to more deeply explore issues and to develop the mother's ability to reflect on the feeding and plan for future feedings.

The purpose of this paper is to describe the implementation of CoReg with mother-very preterm infant dyads during the infant's transition to oral feeding.

\section{Study Design and Methods}

\section{Intervention Description}

While CoReg provides principles of how to feed a VP infant, GP describes the process of working with a mother as she learns how to feed. GP is a method of participatory learning 
structured by a nurse or feeding specialist who guides the mother to extend her feeding practice to incorporate necessary skills and knowledge to become an effective feeding partner for her VP infant (Pridham \& Limbo, 2011). Table 1 lists five processes of GP that are critical components of the intervention.

The intervention involved five sessions, with the first intended to take place prior to when the infant would begin oral feeding, and the four subsequent sessions occurring during the transition to full oral feeding. During the first session we planned to have mothers use the microphone system to listen to their infants' breathing, trial positioning in a semi-elevated, side-lying position, and discuss common feeding strategies required by VP infants. We aimed to play several short video clips of VP infants being fed to sensitize mothers to early infant cues of readiness, breathing interruptions that can be improved by co-regulated pacing, and cues infants provide when they need to rest or end.

Each of the four subsequent feeding sessions focused on feeding-related issues that were identified by the mother or intervention nurse. Each session was organized by the competencies the nurse determined were needed to address these feeding issues. Therefore, each of the four feeding sessions were individualized to the mother and infant with an overarching goal of meeting the infants' and mothers' need to advance their skills. Our goal was to complete all four of the feeding sessions within the first 2 weeks of oral feeding so mothers could refine their skills while their infant remained in the nursery and they continued to have access to nurses and feeding specialists. We offered mothers this plan but also gave them the option to flexibly schedule the four feeding sessions.

\section{Recruitment}

Mothers of VP infants were recruited from a level III neonatal care center in the southeastern United States approximately 2 weeks prior to the infant beginning breast or bottle feeding. This estimate was based on the infant's post-menstrual age (PMA) and health. In the study nursery, infants were offered oral feedings as early as 32 weeks PMA if demonstrating interest in sucking, capacity to maintain an awake state when held, and their need for respiratory support did not exceed use of supplemental oxygen. If the mother planned to breastfeed and could be present in the nursery at least once a day, the first two weeks of oral feeding were preserved for establishment of breastfeeding. After 34 weeks, with exceptions, bottles were offered when the breastfeeding mother was not available.

Mothers were ineligible for the study if they did not speak or read English or were under the age of 18. Infants were ineligible if their gestational age at birth was 31 weeks or greater, or if they had a diagnosed congenital anomaly that could interfere with ingestion, digestion, or elimination. Infants were also ineligible if they had a grade IV intraventricular hemorrhage, were ventilator-dependent beyond 60 days of life, or had not begun oral feeding by 44 weeks PMA. All eligible mothers of eligible infants were identified through chart review and invited to participate in the study. Informed consent was obtained from the infant's mother after the nature of the study procedures had been fully explained. The research protocol and consent forms were approved by the institutional review board at the University of North Carolina at Chapel Hill. All mothers were provided \$20 for travel for each session they participated in. 


\section{Procedures}

For each intervention session, one of two trained intervention nurses arranged to meet with the mother at a time convenient for her and when her infant was scheduled to eat. All intervention sessions were audiotaped for evaluation of fidelity to the intervention by the PI of the study. During the session, the nurse and mother jointly assessed the infant's readiness for oral feeding. If the infant was not ready to eat the session was rescheduled. If ready, a small microphone was secured with gel tape to the infant's neck at the suprasternal notch which transmitted the infants' breathing and swallowing sounds to a video camera and directly to headphones worn by the mother, nurse, and any other participant, such as the father or lactation consultant. These auditory cues provided feedback on the infant's feeding experience and guided the feeding co-regulation. The mother, and at times the father, fed the infant while the nurse provided guidance dependent on the assessed need. In a few instances mothers assumed the role of guide for fathers with the nurse affirming their learning. Following the feeding, the mother and nurse (and fathers, if present) reflected on the feeding as they replayed the videotape, planned refinements for future feedings, and scheduled the next intervention session.

After each session, the intervention nurse completed a structured field note describing the session, including GP methods used, description of the focal issues and competencies that were themes of the session, and facilitators and barriers to implementing the intervention.

\section{Analyses}

Structured field notes, intervention fidelity audiotapes, and feeding session videotapes were the sources of data for evaluation of the intervention's implementation. Content analysis techniques were applied to quantify and describe the implementation of CoReg (Elo \& Kyngas, 2008). A database was constructed to quantify adherence components, including the number, timing and length of the intervention sessions. Details related to facilitators and barriers, such as scheduling issues, were listed for each session. In addition, through a process of reviewing the videotaped feedings and listening to audiotapes of each of the sessions, description of the intervention's delivery were summarized and organized into a matrix of commonly addressed issues and strategies used to guide the development of the mothers' skills. To establish reliability, data from the first three participants were coded by two investigators; a single investigator coded the remaining participants' data.

\section{Results}

\section{Participants}

A total of $61 \mathrm{VP}$ infants were identified from electronic medical records during the 6 month recruitment period. Of these, 22 infants (36.1\%) and their 19 mothers met inclusion criteria. Three of these mothers, including two with sets of twins, declined participation while 16 mothers of 17 infants (77.3\%) enrolled. Tables 2 and 3 present descriptive data on the mothers and their infants. Oral feeding was initiated at a mean PMA of 33.7 weeks (range 32.1-36.9). Eleven infants both breastfed and bottle fed, one infant exclusively breastfed, and five infants exclusively bottle fed. Full oral feeding was reached on average at 37.2 weeks (range 34.6-41.7). 


\section{Success Rate in Completing Sessions}

We had excellent participation in the intervention with retention of all enrolled mothers. Thirteen of the mothers (81.3\%) participated in all five intervention sessions; three mothers completed less than five. One of these mothers was the mother of the set of twins who had difficulty visiting more than a few times a week. To accommodate this mother, we combined the pre-feeding session into a shared session for both infants. She completed a total of seven of the remaining eight sessions. The second mother's infant was transferred to another hospital after the initial session and the third mother's infant progressed to discharge prior to completing the third session.

\section{Timing of the Intervention Sessions}

A total of 74 intervention sessions were conducted, averaging 1 hour in length (range 30-90 minutes). All sessions included the intervention nurse and the mother. A lactation consultant joined for 17 sessions, 10 fathers joined for one to four sessions each, and five sessions included the mother's grandmother or sister.

In review of the intervention field notes, we found that $31 \%$ of the first sessions occurred as planned within the week prior to oral feeding initiation, $25 \%$ occurred earlier than planned (a range of 1.4-3.9 weeks prior to the actual beginning of oral feeding), and 44\% occurred later than planned (from the day of the first oral feeding to 5 days after the first oral feeding). Advance in timing of the first sessions was due to difficulty predicting when infants would be ready for the first oral feeding using their PMA and health. Delay in timing of the first sessions was due to infants developing readiness quicker than expected, or mothers being unable to schedule the session in a timely way.

Eighty percent of mothers chose to spread out the four feeding sessions across a longer period of time than the proposed first 2 weeks of oral feeding. The average span of time was 3.0 weeks with a range of 1.7 to 8.0 weeks, reflecting the infants' wide range of health and the length of the oral feeding transition period. A variety of reasons explained this change in the original plan and provides insight into the realities of learning to feed a VP infant. First, several infants had minimal engagement and therefore, short feeding sessions during the first 2 weeks of oral feeding. Consequently, mothers delayed one to three sessions until the infant was able to engage in feeding for a longer duration so they could experience a greater variety of feeding issues while receiving guidance. Secondly, several mothers were only able to visit once or twice a week and often had difficulty predicting when they would be able to visit, so scheduling was difficult and lengthened the intervention period. Lastly, mothers who were breastfeeding often requested saving one to two intervention sessions until a later time when bottles would be offered. These later sessions focused on bottle feeding techniques so mothers could guide family members or childcare providers to feed once home. In all, six mothers selected all bottle feeding sessions; two mothers selected all breastfeeding sessions, and eight mothers opted for some of each.

\section{Engaging in Guided Participation}

Joint attention of the nurse and mother to the goal of developing the dyads' feeding skills was an essential foundation for each session and a critical component of GP. The nurse 
accomplished this by beginning the session with a discussion of what the mother had learned about her infant since the last session, how her infant had been feeding, what the mother had tried during feeding, and/or what the mother thought the infant and she were currently working on.

Table 4 describes the most frequent feeding issues identified by the mother, the participating family member, or through observation by the intervention nurse. Issues presented as challenges, knowledge gaps, or skills that were needed. These issues became the focus of each session. With each issue, the nurse determined the set of competencies that were needed and used the relationship built with the mother to assess where to begin, given the mother's knowledge base and skill set.

Three main strategies were used to guide the development of the mothers' skills: use of exemplar videos, enhanced audio feedback via the microphone throughout the feedings, and video playback of the feeding. If the first session occurred as planned, prior to the onset of oral feeding, the intervention nurse and mother watched the exemplar feeding video-clips to anticipate future challenges and generate discussion on VP infant feeding. If feedings had already begun by the first session, the exemplar video-clips were used if the intervention nurse introduced an issue that was anticipated but not yet demonstrated by the mother's own infant or to emphasize a specific skill or caregiver strategy. In all, $82.4 \%$ of the dyads viewed the exemplar videos.

The microphone system provided feedback of the infant's capacity to organize swallowing and coordinate breathing with sucking as the infant transitioned from readiness to completion of each feeding. The intervention nurse primarily aimed to help the mother identify breath sounds. We did not plan to focus on swallowing because we thought this might be too complex; however, mothers, on their own, identified swallowing and commented on it, particularly if the infant was breastfeeding. Mothers recognized when swallowing began and linked this to milk letdown, which some could feel, but others were unsure of. Mothers readily understood that breathing and pauses in breathing were important cues. In all, 95.4\% of the feeding sessions included use of the microphone. Lack of use was attributed to equipment error $(n=3)$. Several mothers commented that the idea of using the microphone was the reason they enrolled in the study.

Each session that included a feeding had that portion of the session videotaped. Following the feeding, the videotape was replayed with the mother to reflect on the meaning of the infant's behavior and breathing sounds, how the infant responded to the mother's feeding behaviors, and how future feedings might be improved. In all, $82.8 \%$ of the feeding sessions included video playback. Lack of playback was attributed to equipment error $(n=3)$, mother lacking time ( $n=3)$, or no explanation in field note $(n=5)$. Audiotapes of the intervention sessions provided rich data on the reflective aspect of conducting video playback. It was a time the intervention nurse affirmed the competencies the mother was developing and pointed out ways the infant was showing development and/or stability. Specifically, the nurse commented on mothers' waiting patiently for readiness, reading their infant's cues, providing responsive pacing, being patient and holding still while their infant had sucking pauses and replenished breaths, and stopping to give the infant a rest. When infants showed 
distress or disengagement cues, the nurse often backed up the video to play that portion a second time and invited the mother to explore with her what had happened and what might work better next time. Mothers also asked for sections of the feeding to be replayed to review times they felt uncertain about their infants' behavior or the decisions they had made. During three sessions the nurse replayed a video of the dyad from an earlier session to call attention to and affirm the progress the infant or mother had made.

\section{Facilitation Strategies}

Strategies that facilitated implementation of the intervention were either planned in advance or discovered along the way. The two intervention nurses were trained in (1) early feeding skills assessment of preterm infants (Thoyre \& Shaker, 2011) and using GP in clinical practice (Pridham \& Limbo, 2011). The nurses worked together to refine the intervention with the first two enrolled mothers. Six mothers were subsequently guided solely by one of the nurses and the remaining nine mothers had sessions split between them. The study team had on-going supervision meetings to discuss active cases, review recently completed videotapes and come to consensus on providing GP. With these strategies, the consistency of the delivery of the intervention was optimized.

We learned early on that mothers needed a high degree of flexibility in scheduling the sessions. Mothers were often working or in school and caring for other small children at home. Their lives were busy with competing demands for their time and resources. In addition, infants could not be counted on to be ready to eat when mothers visited. To ease mothers' burdens we provided reimbursement for travel expenses, agreed to meet 7 days a week during the day or evening hours, and rescheduled sessions if the infant was not ready to eat.

\section{Barriers to Implementation}

There were several barriers to implementing CoReg. For one-third of the feeding sessions, infants were either not ready to eat or able to eat for only a short time. Infants' health precluded oral feeding altogether for four of the scheduled sessions. Mother's health and weather events also led to difficulty scheduling and cancellation of sessions. Some mothers were unable to visit more than once or twice a week. While we scheduled sessions during these infrequent visits, if mothers could only stay for a short time the intervention competed with the need for other health care providers' attention, particularly as discharge became imminent. Finally, despite conducting the study in a nursery with a similar approach to feeding preterm infants as the study used, we found at times, mothers were given inconsistent or divergent ideas about how to feed their infants. While a challenge for the intervention nurse, this was most difficult for the mothers who were trying to learn a unique style of feeding and gather as much information as they could from those around them, while needing to trust what was being offered by those caring for their infants. Learning the complexities of feeding a VP infant can be more difficult when providers are not in agreement on how to feed. 


\section{Clinical Nursing Implications}

Nurses guide parents and preterm infants in learning to feed during the final weeks of hospitalization. This necessitates having the ability to identify and understand the meaning of preterm infants' adaptive feeding behaviors and stress responses, and understanding of the emergence of infants' skill at orientating and sustaining attention, preserving energy, modulating stimulation, and coordinating sucking and swallowing with breathing. Just as critical to understanding the infant, nurses need to be effective at guiding parents who are early in learning to protect and enhance their infants' feeding experiences. This study examined the implementation of CoReg with mothers of VP infants, which included five sessions of guided feeding support with enhanced audio feedback of breathing and swallowing cues, video review of the just completed feeding, and joint planning with an intervention nurse to optimize future feedings.

The implementation of CoReg would be enhanced if the nurse who is caring for the infant acted as the guide since they would naturally be available when mothers' visits coincide with their infants' readiness to eat. The process of providing GP is consistent with how nurses help parents learn to care for children under novel circumstances (Pridham, Limbo, Schroeder, Thoyre, \& Van Riper, 1998). It is critical that neonatal care units support nurses in having time for this important activity. Caregiving is socially constructed within the culture of a family and community; with VP infant feeding, this community is the neonatal care unit. The unit, and how it approaches feeding, therefore, is a highly influential springboard for how parents come to view feeding and their role as a feeding partner.

The approach of GP offers a promising new direction for nurses to use with mothers learning to co-regulate their VP infants' feedings. An important part of participatory learning is arrangement and organization of the mother's experience so she has opportunities for learning. Watching "how-to" videos or reading printed guidelines will not be sufficient. A mother needs to experience what it feels like to feed her infant and encounter the types of decisions that arise while a more knowledgeable "guide" helps her make sense of what she is coming to know about feeding and affirms her learning. When a nurse develops a relationship with the mother, sits with her while she feeds, and, through conversation and action, establishes a partnership with her as she feeds, optimal feeding can be accomplished. For example, the nurse may state, "Since you've noticed your baby gets tired easily with feeding, let's see what happens if we provide rest periods, starting in the first minute of the feeding. A rest means you'll ease the nipple out and, without movement of your baby, allow your baby's breathing to calm. I'll let you know when we should give a rest, okay?" In this example, the nurse uses her advanced knowledge that providing rests early in the feeding may lengthen the infant's endurance. Partnering with the mother during the physical act of resting allows the mother to experience giving the rest, without having to make the decision about when to provide it. In this study, mothers and fathers expressed satisfaction with this level of guidance.

In GP, the nurse determines where parents are in their learning and supplies support as needed. Essentially, in parallel, the nurse co-regulates the dyad's feeding. Since the nurse needs to decide where to begin, there is much to be learned from the mother and from 
observation of the mother and infant feeding. As an illustration, if a mother describes the challenge she is having figuring out when to provide co-regulated pacing, and the nurse observes that the mother is also not yet able to provide consistent postural support for her infant during feeding, the nurse recognizes that these are both novel motor patterns for mothers. To facilitate the mother's learning of both co-regulated pacing and postural support, the nurse can physically reach out and stabilize the infant's position and remain in contact with the infant as long as it is needed, while guiding the mother's pacing. This frees the mother to focus on the co-regulatory skills she is learning, and in the process, the nurse models the level of postural support the mother will need to incorporate into her care. As mothers become familiar with what is needed, they begin to do it on their own and the nurse's role shifts to affirming learning and providing less direct physical support.

\section{Acknowledgments}

Sources of funding: This research was supported by the National Institutes of Health, National Institute of Nursing Research: R21NR012507 (Thoyre) and T32 NR007091 (McKecknie).

\section{References}

DeMauro SB, Patel PR, Medoff-Cooper B, Posencheg M, Abbasi S. Postdischarge feeding patterns in early- and late-preterm infants. Clinical Pediatrics. 2011; 50(10):957-962. DOI: 10.1177/0009922811409028 [PubMed: 21856965]

Dodrill P, Donovan T, Cleghorn G, McMahon S, Davies PS. Attainment of early feeding milestones in preterm neonates. Journal of Perinatology. 2008; 28(8):549-555. DOI: 10.1038/jp.2008.56 [PubMed: 18580883]

Elo S, Kyngas H. The qualitative content analysis process. Journal of Advanced Nursing. 2008; 62(1): 107-115. DOI: 10.1111/j.1365-2648.2007.04569.x [PubMed: 18352969]

Gewolb IH, Bosma JF, Reynolds EW, Vice FL. Integration of suck and swallow rhythms during feeding in preterm infants with and without bronchopulmonary dysplasia. Developmental Medicine \& Child Neurology. 2003; 45(5):344-348. DOI: 10.1111/j.1469-8749.2003.tb00406.x [PubMed: 12729149]

Gewolb IH, Vice FL. Maturational changes in the rhythms, patterning, and coordination of respiration and swallow during feeding in preterm and term infants. Developmental Medicine \& Child Neurology. 2006; 48(7):589-594. DOI: 10.1111/j.1469-8749.2006.tb01320.x [PubMed: 16780629]

Goldfield EC. A dynamical systems approach to infant oral feeding and dysphagia: From model system to therapeutic medical device. Ecological Psychology. 2007; 19(1):21-48. DOI: $10.1080 / 10407410701290791$

Hjalmarson O, Brynjarsson H, Nilsson S, Sandberg KL. Persisting hypoxaemia is an insufficient measure of adverse lung function in very immature infants. Archives of Disease in Childhood Fetal and Neonatal Edition. 2014; 99(4):F257-262. DOI: 10.1136/archdischild-2013-304625 [PubMed: 24356176]

Hwang YS, Ma MC, Chen-Sea MJ, Kao HM, Tsai WH. Factors affecting early feeding performance in preterm infants below 32 weeks gestation. Journal of Tropical Pediatrics. 2012; 58(1):77-78. DOI: 10.1093/tropej/fmr008 [PubMed: 21292741]

Jobe AH, Bancalari E. Bronchopulmonary dysplasia. American Journal of Respiratory and Critical Care Medicine. 2001; 163(7):1723-1729. DOI: 10.1164/ajrccm.163.7.2011060 [PubMed: 11401896]

Jonsson M, van Doorn J, van den Berg J. Parents' perceptions of eating skills of pre-term vs full-term infants from birth to 3 years. International Journal of Speech-Language Pathology. 2013; 15(6): 604-612. DOI: 10.3109/17549507.2013.808699 [PubMed: 24007388] 
Kirk AT, Alder SC, King JD. Cue-based oral feeding clinical pathway results in earlier attainment of full oral feeding in premature infants. Journal of Perinatology. 2007; 27(9):572-578. DOI: 10.1038/sj.jp.7211791 [PubMed: 17625573]

Maitre NL, Ballard RA, Ellenberg JH, Davis SD, Greenberg JM, Hamvas A, Pryhuber GS. Respiratory consequences of prematurity: evolution of a diagnosis and development of a comprehensive approach. Journal of Perinatology. 2015; 35(5):313-321. DOI: 10.1038/jp.2015.19 [PubMed: 25811285]

McCain GC, Del Moral T, Duncan RC, Fontaine JL, Pino LD. Transition from gavage to nipple feeding for preterm infants with bronchopulmonary dysplasia. Nursing Research. 2012; 61(6): 380-387. DOI: 10.1097/NNR.0b013e318268cefb [PubMed: 22960584]

Migraine A, Nicklaus S, Parnet P, Lange C, Monnery-Patris S, Des Robert C, Roze JC. Effect of preterm birth and birth weight on eating behavior at $2 \mathrm{y}$ of age. American Journal of Clinical Nutrition. 2013; 97(6):1270-1277. DOI: 10.3945/ajcn.112.051151 [PubMed: 23615831]

Mizuno K, Nishida Y, Taki M, Hibino S, Murase M, Sakurai M, Itabashi K. Infants with bronchopulmonary dysplasia suckle with weak pressures to maintain breathing during feeding. Pediatrics. 2007; 120(4):e1035-1042. DOI: 10.1542/peds.2006-3567 [PubMed: 17893188]

Pridham, K., Limbo, R. Guided Participation Certificate Program. University of Wisconsin-Madison, School of Nursing; Madison, WI: 2011.

Pridham KF, Limbo R, Schroeder M, Thoyre S, Van Riper M. Guided participation and development of care-giving competencies for families of low birth-weight infants. Journal of Advanced Nursing. 1998; 28(5):948-958. [PubMed: 9840866]

Puckett B, Grover VK, Holt T, Sankaran K. Cue-based feeding for preterm infants: A prospective trial. American Journal of Perinatology. 2008; 25(10):623-628. DOI: 10.1055/s-0028-1090583 [PubMed: 18841533]

Reyna BA, Pickler RH, Thompson A. A descriptive study of mothers' experiences feeding their preterm infants after discharge. Advances in Neonatal Care. 2006; 6(6):333-340. DOI: 10.1016/ j.adnc.2006.08.007 [PubMed: 17208164]

Ross ES, Browne JV. Feeding outcomes in preterm infants after discharge from the Neonatal Intensive Care Unit (NICU): A systematic review. Newborn \& Infant Nursing Reviews. 2013; 13(2):87-93. DOI: 10.1053/j.nainr.2013.04.003

Ross ES, Philbin MK. Supporting oral feeding in fragile infants: an evidence-based method for quality bottle-feedings of preterm, ill, and fragile infants. Journal of Perinatal and Neonatal Nursing. 2011; 25(4):349-357. quiz 358-349. DOI: 10.1097/JPN.0b013e318234ac7a [PubMed: 22071619]

Sables-Baus, S., DeSanto, K., Henderson, S., Kunz, JL., Morris, AC., Shields, L., McGrath, JM. Infant-directed oral feeding for premature and critically ill hospitalized infants: Guideline for practice. Chicago, IL: National Association of Neonatal Nurses; 2013.

Stevens EE, Gazza E, Pickler R. Parental experience learning to feed their preterm infants. Advances in Neonatal Care. 2014; 14(5):354-361. DOI: 10.1097/ANC.0000000000000105 [PubMed: 25000100]

Thoyre S, Holditch-Davis D, Schwartz T, Melendez CR, Nix B. Coregulated approach to feeding preterm infants with lung disease: Effects during feeding. Nursing Research. 2012; 62(2):242-251. DOI: 10.1097/NNR.0b013e31824b02ad

Thoyre S, Park J, Pados B, Hubbard C. Developing a co-regulated, cue-based feeding practice: The critical role of assessment and reflection. Journal of Neonatal Nursing. 2013; 19(4):139-148. DOI: 10.1016/j.jnn.2013.01.002 [PubMed: 25814831]

Thoyre, S., Shaker, C. The Early Feeding Skills (EFS) assessment tool: A guide to cue-based feeding in the NICU. Continuing Education Course provided by Pediatric Resources, Inc; Boston, MA: 2011.

Wang LY, Luo HJ, Hsieh WS, Hsu CH, Hsu HC, Chen PS, Jeng SF. Severity of bronchopulmonary dysplasia and increased risk of feeding desaturation and growth delay in very low birth weight preterm infants. Pediatric Pulmonology. 2010; 45(2):165-173. DOI: 10.1002/ppul.21171 [PubMed: 20054861] 


\section{Call outs}

1. Parents of very preterm infants need to be skilled in providing co-regulated, cue-based feeding.

2. The skills parents need to co-regulate feedings of VP infants are novel and non-intuitive.

3. Nurses need to be effective at guiding parents, who are early in learning, to protect their infants' feeding experiences.

4. The neonatal care unit's approach to feeding is foundational for how parents come to view feeding and their role as a feeding partner.

5. When guiding mothers to feed their preterm infants the nurse needs to decide where to begin; consequently, there is much to learn from the mother and from observation of the mother and infant together. 


\section{Box}

\section{Suggested Clinical Nursing Implications}

- Understand that throughout the entire transition to oral feeding, mothers want and support nursing observation and assessment of their skills.

- $\quad$ Be present with mothers as they are learning novel feeding behaviors and non-intuitive ways of thinking about feeding their infant.

- Make explicit the approach that is required. It is not black and white; rather, it is a process of observation, giving meaning, reflecting, planning, and refining.

- $\quad$ Consider using the mother's smart phone to videotape challenging segments of the feeding, such as getting started, or deciding when to end. With guidance, mothers can learn to use the video to reflect on how feeding might go more smoothly next time.

- Make adequate breathing and remaining calm the feeding outcomes of highest importance.

- $\quad$ Affirm the mother's learning; help to create her sense of self as doing her very best for her infant; this will strengthen the parent-child feeding relationship. 


\section{Table 1}

\section{Essential Processes of Guided Participation for Development of Mothers' Feeding Skills}

Establishing joint attention with the mother about what the guide and mother will be doing together

2 Arranging opportunities for the guide and the mother to learn about the dyad's salient feeding issues

3 Maintaining the mother's engagement in learning by focusing on salient issues and the competencies that are needed to address them

$4 \quad$ Guiding the mother to make connections between what she is coming to know about her infant and how her infant responds to feeding and to her feeding interventions

$5 \quad$ Guiding the mother toward a sense of self as central to and capable of guiding her infant's future feedings 
Table 2

Demographic Characteristics of Participating Mothers ( $\mathrm{n}=16$ )

\begin{tabular}{llc}
\hline \multicolumn{3}{c}{ Characteristic: Mean (SD) or \% } \\
\hline Education (years) & & $16.0(4.1)$ \\
Age (years) & & $29.4(5.9)$ \\
Currently employed & & $50.0 \%$ \\
First Time Mother & & $47.0 \%$ \\
Two Parent Family & & $100 \%$ \\
Race/ethnicity & White, non-Hispanic & $37.5 \%$ \\
& Black, non-Hispanic & $37.5 \%$ \\
& Hispanic & $18.8 \%$ \\
& More than one & $6.2 \%$ \\
& $<29,999 /$ year & $13.3 \%$ \\
Family Income & \\
& $30-49,999 /$ year & $33.3 \%$ \\
& $>50,000 /$ year & $53.3 \%$ \\
Missing data; $n=15$. & &
\end{tabular}

Missing data; $n=15$. 
Table 3

\begin{tabular}{|c|c|c|}
\hline \multicolumn{3}{|c|}{ Characteristic: Mean (SD) or \% } \\
\hline GA at birth (weeks) & & $27.9(2.3)$ \\
\hline Birth weight (g) & & $1037(323)$ \\
\hline Male & & $41.2 \%$ \\
\hline SGA & & $17.6 \%$ \\
\hline NEC & & $11.8 \%$ \\
\hline \multirow[t]{4}{*}{ Lung Disease } & None & $35.3 \%$ \\
\hline & Mild & $17.6 \%$ \\
\hline & Moderate & $29.4 \%$ \\
\hline & Severe & $17.6 \%$ \\
\hline Grade III IVH & & $5.9 \%$ \\
\hline PMA at Discharge (weeks) & & $38.4(2.0)$ \\
\hline DOL at Discharge & & $72.8(26.5)$ \\
\hline
\end{tabular}

Note: $\mathrm{GA}=$ gestational age; $\mathrm{SGA}=$ small for gestational age; $\mathrm{NEC}=$ necrotizing enterocolitis; IVH=intraventricular hemorrhage; PMA=postmenstrual age; $\mathrm{DOL}=$ day of life. 
Table 4

Most frequent feeding issues identified and related competencies to be developed

\begin{tabular}{|c|c|}
\hline Issue identified & Competencies needed to resolve the issue \\
\hline $\begin{array}{l}\text { "Reading" cues of calmness, } \\
\text { organization, engagement, stress, and } \\
\text { disengagement }\end{array}$ & $\begin{array}{l}\text { - } \\
\text { - } \\
\text { - } \\
\text { - } \\
\text { Accurate interpretation of the meaning of preterm infant behaviors } \\
\text { Confidence to respect infant's limits }\end{array}$ \\
\hline Co-regulating sufficient breaths & $\begin{array}{l}\text { Understanding why VP infants are at risk for insufficient breathing and the impact } \\
\text { this has on feeding (fatigue, early cessation, physiologic instability, stress) } \\
\text { - } \quad \text { Physical skill at shifting the infant from sucking to breathing and back to sucking } \\
\text { - Ability to explore the need for and timing of providing breathing co-regulation }\end{array}$ \\
\hline Providing motoric stability & $\begin{array}{l}\text { - Understanding the value of postural support } \\
\text { - } \quad \text { Physical skill at swaddling and holding the infant with neutral head/neck alignment }\end{array}$ \\
\hline Regulating milk flow & $\begin{array}{l}\text { Understanding the potential impact of milk flow on the infant's feeding behavior } \\
\text { (strength of sucking, length of sucking burst, contact of the nipple on the tongue, milk } \\
\text { spill, threat of aspiration) } \\
\text { Ability to evaluate the match of the infant's capacity to manage milk flow with the } \\
\text { feeding system (breast, bottle, various nipple systems) }\end{array}$ \\
\hline Providing rest periods & $\begin{array}{l}\text { - Understanding the connection of providing brief }(30-60 \mathrm{sec}) \text { rest periods for } \\
\text { replenishment of breath with the infant's capacity for feeding endurance } \\
\text { Physical skill and timing for removing the nipple, and quietly, without movement, } \\
\text { structuring an opportunity to replenish breaths and restore }\end{array}$ \\
\hline $\begin{array}{l}\text { Determining readiness, preparing the } \\
\text { infant, and allowing time for full } \\
\text { organization }\end{array}$ & $\begin{array}{l}\text { - Understanding the value of helping the infant to become fully organized and ready for } \\
\text { feeding } \\
\text { Physical skill at rooting and waiting for organization prior to placing the nipple }\end{array}$ \\
\hline $\begin{array}{l}\text { Monitoring and providing appropriate } \\
\text { stimulation; preserving energy }\end{array}$ & $\begin{array}{l}\text { - Understanding the sensitivity of VP infants to stimulation and the need for neuro- } \\
\text { protection } \\
\text { - }\end{array}$ \\
\hline
\end{tabular}

